\title{
Factores psicológicos asociados a la vulnerabilidad de lesiones. Estudio de caso en un equipo de fútbol semi-profesional
}

\author{
Patricia Catalá y Cecilia Peñacoba \\ Universidad Rey Juan Carlos, España
}

RESUMEN: El presente trabajo analiza la asociación entre determinadas características psicológicas de los deportistas y la ocurrencia de lesiones en un equipo de fútbol de tercera división ( $n=22)$. En particular, se valoró la posible influencia de la ansiedad (CSAI-2), las variables relacionadas con el rendimiento deportivo (CPRD), las estrategias de afrontamiento (ACSQ-1) y la regulación emocional (IE). Se llevó a cabo un registro de lesiones durante los 6 meses posteriores a la evaluación. Se observan puntuaciones significativamente más elevadas en inteligencia emocional, reestructuración cognitiva, autoconfianza y en variables relacionadas con el rendimiento deportivo, así como menores puntuaciones en retraimiento mental y conductas de riesgo en los jugadores sin lesión. Se concluye que ciertas variables psicológicas se relacionan con la ausencia de lesiones deportivas, por lo que recomienda su evaluación y el desarrollo de programas de intervención psicológica para su prevención.

PALABRAS CLAVES: estrategias de afrontamiento, inteligencia emocional, características psicológicas de rendimiento, lesión deportiva, fútbol.

\section{Psychological factors associated with the vulnerability to injuries in soccer players. A case report in a semi-professional soccer team}

ABSTRACT: The association between certain psychological characteristics of athletes and incidence of injuries in a third division soccer team is analyzed $(n=22)$. In particular, the potential influence of anxiety (CSAI-2), variables related to sports performance (CPRD), coping strategies (ACSQ-1), and emotional regulation (EI) was assessed. Injuries sustained during the 6 months following the evaluation were recorded. Players with no injuries had significantly higher scores in emotional intelligence, cognitive restructuring, selfconfidence, and variables related to sports performance, and lower scores in mental withdrawal and risk behaviors. It is concluded that some psychological variables are related to absence of sports injuries. Evaluation of such variables and development of psychological intervention programs for their prevention are therefore recommended.

KEYWORDS: coping strategies, emotional intelligence, psychological performance characteristics, sports injuries, soccer.

\section{Fatores psicológicos associados à vulnerabilidade de lesões. Estudo de caso numa equipa de futebol semiprofissional}

RESUMO: O presente trabalho analisa a associação entre certas características psicológicas dos atletas e a ocorrência de lesões numa equipa de futebol da terceira divisão $(n=22)$. Em particular, foram avaliadas a possível influência da ansiedade (CSAl-2), as variáveis relacionadas com o desempenho desportivo (CPRD), as estratégias de adaptação (ACSQ-1) e a regulação emocional (EI). Foi realizado um registo de lesões durante os 6 meses após a avaliação. Foram observadas pontuações significativamente mais altas em inteligência emocional, reestruturação cognitiva, autoconfiança e em variáveis relacionadas com o desempenho desportivo, bem como

\footnotetext{
Patricia Catalá iD https://orcid.org/0000-0003-4989-9099 es psicóloga y profesora, Departamento de Psicología. Universidad Rey Juan Carlos. Cecilia Peñacoba iD https://orcid.org/0000-0001-6307-5921 es psicóloga y profesora, Departamento de Psicología. Universidad Rey Juan Carlos.

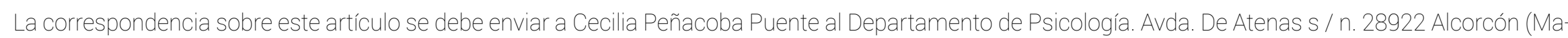
drid). España. E-mail:
} 
pontuações mais baixas em retraimento mental e comportamentos de risco em jogadores sem lesões. Conclui-se que determinadas variáveis psicológicas estão relacionadas com a ausência de lesões desportivas, razão pela qual se recomenda a sua avaliação e o desenvolvimento de programas de intervenção psicológica para a sua prevenção.

PALAVRAS-CHAVE: estratégias de adaptação, inteligência emocional, características psicológicas do desempenho, lesões desportivas, futebol.

Algunos estudios indican desde hace décadas los altos índices de lesiones en el deporte (Pujals et al., 2016); en particular, el fútbol es considerado uno de los deportes que mayor riesgo de lesión presenta (Llana, Pérez y Lledó, 2010). En Europa es responsable de entre un cuarto y la mitad de las lesiones que se registran (Tscholl et al., 2007). Debido a las consecuencias tanto de índole económico, como sobre la salud de los deportistas, la lesión en el fútbol supone un tema de máxima preocupación (Padegimas et al., 2016). Se ha constatado que la lesión, además del evidente daño físico que provoca, supone una situación con importantes consecuencias psicológicas tanto para el deportista como para su entorno social (Buceta, 2008).

Pese a las investigaciones realizadas en este campo, hasta el momento no se ha podido consensuar una definición universal de lesión deportiva (Palmi, 2014). A modo de ejemplo, Hinrichs (1995) define la lesión como "un término general de aplicación a todos los procesos que destruyen o alteran la integridad de un tejido o parte orgánica". Atendiendo a las sugerencias de diversos autores (Buceta, 1996; Pascual y Aragües, 1998), podemos describir las lesiones en función del nivel de gravedad: leve (precisa tratamiento y al menos un día de recuperación), moderada (necesita tratamiento, suponiendo seis días de recuperación), grave (requiere baja deportiva de entre uno y tres meses) y muy grave (implica baja deportiva de al menos cuatro meses, requiere intervención quirúrgica e incluso rehabilitación mantenida). Son diversos los factores, de carácter extrínseco e intrínseco, los que marcan la etiología de estas lesiones. Mientras que los factores de riesgo intrínseco se relacionan con las características biológicas o psicosociales del deportista, los factores de riesgo externo se relacionan con la metodología de entrenamiento (Eils et al., 2004).

En relación a las características intrínsecas, cada vez son más numerosos los estudios que tienen por objetivo analizar la relación entre las variables psicológicas y la lesión deportiva, puesto que los propios deportistas son conscientes de la influencia que tienen las características psicológicas como factor causal de las mismas (Herring et al., 2017; Schinke et al., 2017; Soligard, et al., 2016).

Andersen y Williams (1988) propusieron un influyente modelo explicativo de la relación entre factores psicológicos y lesión deportiva, en el que el concepto principal es el estrés. Este modelo de estrés y lesión deportiva hipotetiza que un deportista ante una situación estresante emite una respuesta (denominada de estrés) producto de la valoración cognitiva que hace de aquélla, provocando cambios fisiológicos (incremento de la tensión muscular) y atencionales (focalización inadecuada de la atención) que aumentan la probabilidad de lesionarse. Además, otros componentes del modelo (personalidad, historia de estrés del deportista y los recursos de afrontamiento) mediarán en el carácter de la respuesta, potenciando el estrés o ayudando a controlarlo (Abenza et al., 2009; Garcia-Mas et al., 2014).

En base a este modelo general, Olmedilla y García-Mas (2009) elaboraron un modelo global de las lesiones deportivas donde destacaron cinco líneas principales de investigación. La primera, estudia la relación entre los factores psicológicos y la vulnerabilidad de los deportistas a lesionarse; la segunda, analiza la percepción de los deportistas sobre la importancia del factor psicológico como causante de la lesión; la tercera valora la relación entre la historia de lesiones de los deportistas y su influencia sobre diferentes variables psicológicas de los mismos; la cuarta, estudia las reacciones emocionales y psicológicas ante la lesión. Finalmente, la quinta, desde una perspectiva aplicada, analiza los programas de intervención psicológica dirigidos tanto a la lesión deportiva (tanto a nivel preventivo como rehabilitador) como a la psicoeducación de deportistas y entrenadores (Mugele et al., 2019).

Este modelo, a su vez, organiza las variables psicológicas en función de tres ejes de análisis: eje causal (antecedentes y consecuentes), eje temporal (distribución en función del tiempo) y eje conceptual (modelos teóricos relevantes).

Las principales variables psicológicas han sido estudiadas, en la mayoría de las ocasiones, en función del eje temporal. Así, han sido establecidas diferentes etapas: previas a la lesión deportiva, vigentes durante el transcurso de la misma y presentes una vez curada la lesión. De las anteriores etapas, merecen destacarse los trabajos que se centran en las variables implicadas en la vulnerabilidad del deportista a lesionarse (es decir, anteriores a la lesión), como la autoconfianza (Berengüí y Puga, 2015; Gonzélez-Reyes et al., 2017), 
la ansiedad competitiva (Prieto y Olmedilla, 2015; Zurita et al., 2017), el estrés psicosocial (Olmedilla et al., 2018; Olmedilla et al., 2014; Zurita et al., 2014; Zurita-Ortega, et al., 2017), la motivación (Kerr et al., 2004; Prieto et al., 2015), los recursos de afrontamiento (Gonzales-Reyes et al., 2017; Ríos et al., 2019), el empleo de las conductas de riesgo (Fuller, 2005; Tuner et al., 2004), los procesos emocionales (Berengüí et al., 2013; Rubio et al., 2014) y el estado de ánimo (García y Más, 2011; Rozen y Horne, 2007).

En este contexto, el objetivo del presente trabajo, empleando un diseño de caso, pretende analizar los factores psicológicos asociados con la aparición de lesiones deportivas en jugadores de fútbol, situándose, por lo tanto, dentro del eje temporal anterior a la lesión deportiva (vulnerabilidad a la lesión). Se han incorporado dentro de las variables de vulnerabilidad, y atendiendo a la literatura previa, la ansiedad y autoconfianza, las estrategias de afrontamiento, determinadas características asociadas al rendimiento deportivo y la inteligencia emocional. Se establece como hipótesis que los jugadores que se lesionan tendrán puntuaciones más altas en estrategias de afrontamiento, concretamente en conductas de riesgo y retraimiento mental, y puntuaciones más bajas en regulación emocional, autoconfianza, motivación y control de estrés.

\section{Método}

\section{Participantes}

La muestra estaba formada por 22 jugadores de fútbol masculino pertenecientes a un club de la tercera división de Madrid, con un rango de edad entre 19 y los 30 años ( $M=$ 23.31, D. $T=4.24)$. La media de años que llevaban practicando este deporte es de $17.54(D . T=4.64)$ y dentro de esta misma categoría 3.31 años (D.T = 3.06). El 40.9\% de los jugadores habían tenido alguna lesión (ya sea leve, moderada o grave) en los últimos 6 meses.

\section{Variables e instrumentos}

A todos los futbolistas, un psicólogo del deporte les administró un protocolo de autoinforme donde se recogían los siguientes cuestionarios:

Inventario de Ansiedad Estado Competitiva 2 (Competitive State Anxiety Inventory 2, CSAI- 2, Martens et al., 1990)

Se empleó la versión española de Capdevila (1997). Este cuestionario consta de 27 ítems que evalúan tres factores: estado de ansiedad cognitiva (9 ítems), estado de ansiedad somática (9 ítems) y autoconfianza (9 ítems) con un formato de respuesta tipo Likert de cuatro puntos. Los deportistas responden a cada uno de los ítems partiendo de la consigna general: "Antes de la competición...". La puntuación total de cada dimensión se obtiene del promedio de las puntuaciones de sus ítems, oscilando, por lo tanto, el rango teórico de cada dimensión entre 1 y 4, indicando mayores puntuaciones una mayor intensidad en la variable en cuestión. Capdevila (1997) encontró valores Alpha de Cronbach de .91 para ansiedad cognitiva, .93 para ansiedad somática y .90 para autoconfianza. En el presente estudio, los Alpha de Cronbach para cada una de las dimensiones fueron: .85 para ansiedad cognitiva, .84 para ansiedad somática y .72 para autoconfianza.

\section{Estrategias de afrontamiento en el deporte}

Se empleó el Cuestionario de Aproximación al Afrontamiento en el Deporte (Approach to Coping in Sport Questionnaire, ACSQ-1) en su versión española (Kim et al., 2003). El cuestionario consta de 28 ítems en los que el deportista debe responder en una escala Likert de 5 puntos la alternativa que indique con qué frecuencia usa ciertas estrategias de afrontamiento en las situaciones competitivas. El instrumento permite la evaluación de cinco estrategias de afrontamiento: calma emocional (7 ítems), reestructuración cognitiva (6 ítems), retraimiento mental (6 ítems), conductas de riesgo (4 ítems) y búsqueda de apoyo social (5 ítems) en un rango teórico de 1 a 5 , indicando mayores puntuaciones un mayor empleo de la estrategia de afrontamiento. Kim et al. (2003) encontraron valores Alpha de Cronbach de .71 para calma emocional, .74 para reestructuración cognitiva, .78 para retraimiento mental, .64 para conductas de riesgo y .78 para búsqueda de apoyo social. En el presente estudio, se obtuvo una consistencia interna del coeficiente Alfa de Cronbach de .71 para calma emocional, de .74 para reestructuración cognitiva, de .78 para retraimiento mental, de .64 para conductas de riesgo y de .78 para búsqueda de apoyo social.

\section{Características Psicológicas Relacionadas con el Rendimiento Deportivo (CPRD) (Gimeno et al., 2001)}

En su conjunto, el instrumento pretende valorar los factores psicológicos que pueden afectar al rendimiento. Consta de 55 ítems con un formato de respuesta tipo Likert de 6 puntos que permite la evaluación de cinco dimensiones: control del estrés, influencia de la evaluación del rendimiento, 
motivación, habilidad mental y cohesión de equipo. Se convirtieron las puntuaciones directas a puntuaciones normativas de acuerdo a los baremos establecidos en muestra española (Gimeno et al., 2001). Gimeno et al. (2001) encontraron valores de Alfa de Cronbach de 88 para control del estrés, .72 para influencia de la evaluación del rendimiento, .67 para motivación, .34 para habilidad mental y .78 para cohesión de equipo. En el presente estudio, se ha obtenido un coeficiente Alfa de Cronbach de .92 para control del estrés, .78 para influencia de la evaluación del rendimiento, .80 para motivación, 90 para habilidad mental y .70 para cohesión de equipo.

\section{Inteligencia emocional}

Se empleó la versión validada en deportistas por García et al. (2013) del Inventario de Inteligencia Emocional de Schutte et al. (1998). Este instrumento consta de 28 ítems ofreciendo una estructura de cuatro dimensiones: percepción emocional (8 ítems), gestión auto-emocional (8 ítems), gestión hetero-emocional (10 ítems) y utilización emocional (4 ítems). Los ítems tienen una escala de respuesta tipo Likert de 5 puntos, siendo el 1 Totalmente en desacuerdo y el 5 Totalmente de acuerdo. Mayor puntuación en la dimensión indica un mejor manejo de la habilidad. Los valores de Alpha de Cronbach encontrados por García et al. (2013) fueron de .77 en percepción emocional, .77 en gestión auto-emocional, .78 en gestión hetero-emocional y .63 en utilización emocional. En el presente estudio, los Alphas de Cronbach encontrados fueron: .84 para percepción emocional, .89 para gestión auto-emocional, .90 para gestión hetero-emocional y .86 para utilización emocional.

Para la evaluación y seguimiento de las lesiones deportivas se utilizó una hoja de registro (Olmedilla et al., 2011, adaptada de Díaz, 2001) que cumplimentaban los fisioterapeutas del equipo de fútbol participante.

\section{Diseño y procedimiento}

Se empleó un diseño de investigación de estudio de caso (Cozby y Pineda, 2005), concretamente de un equipo de fútbol, prospectivo. El hecho de trabajar con un solo equipo, pese a la limitación asociada de la escasez de la muestra, se debe fundamentalmente a la necesidad de controlar las variables extrínsecas a la lesión, vinculadas a la metodología de entrenamiento (Eils et al., 2004). Igualmente, se optó por la selección de un equipo que no hubiera trabajado antes con psicólogos deportivos a nivel individual ni grupal para evitar el posible sesgo producido por el efecto de la interven- ción psicológica sobre las variables psicosociales vinculadas a la lesión (Zurita et al., 2014).

Respecto a su naturaleza prospectiva, el estudio contó con dos momentos de evaluación. En el primer momento, se procedió a aplicar el protocolo de evaluación anteriormente descrito a los jugadores del equipo de fútbol. A partir de la recogida de estas variables psicosociales, se procedió, durante el segundo momento temporal (seis meses), al registro de las lesiones que padecían los jugadores. Una vez finalizados estos seis meses, se procedió a contabilizar el número de lesiones y su localización para cada uno de los jugadores durante el periodo establecido. Posteriormente se clasificaron según su nivel de gravedad (leve, moderado, grave o muy grave).

Respecto al procedimiento para la realización del estudio, se solicitó permiso al responsable del club. Una vez obtenido, se procedió a la firma del correspondiente consentimiento informado por parte de cada uno de los jugadores y a la administración de los cuestionarios de autoinforme en una sala que reunía las condiciones necesarias para su correcto uso (luz, ausencia de ruido, buena temperatura y espacio para recibir a los deportistas. La evaluación fue realizada por un psicólogo deportivo de forma individualizada y tuvo una duración de 20 minutos.

El presente estudio recibió la aprobación del Comité ético de la Universidad Rey Juan Carlos (número de registro: 190720167016).

\section{Análisis estadísticos}

Los análisis se realizaron con el programa estadístico SPSS 21 (IBM, 2012). Se llevaron a cabo análisis descriptivos y de consistencia interna (coeficiente Alpha de Cronbach). Para analizar las posibles diferencias existentes entre las variables psicológicas de interés y el hecho de si el jugador había tenido o no lesión, se procedió a la realización de la prueba no paramétrica $U$ de Mann-Whitney.

\section{Resultados}

\section{Descripción cualitativa y cuantitativa del número de lesiones}

En la Tabla 1 puede observarse los jugadores y tipo de lesión experimentada por cada jugador $(n=9)$. Nueve de los veintidós jugadores (40.9\%) habían experimentado una lesión en el momento temporal establecido (seis meses). Atendiendo a su gravedad (Buceta, 1996; Pascual y Marcos, 
Tabla 1. Descripción del tipo de lesión por jugador de fútbol $(n=9)$

Lesión (gravedad)

\begin{tabular}{|c|c|}
\hline Jugador 1 & Problemas de espalda (leve) \\
\hline Jugador 2 & Edema óseo (moderada) \\
\hline Jugador 3 & Pubalgia (grave)/ $2^{\mathrm{a}}$ lesión Rotura de abductor (moderada) \\
\hline Jugador 4 & Esguince de tobillo (moderada) \\
\hline Jugador 5 & Edema óseo (moderada) \\
\hline Jugador 6 & Principio de pubalgia (moderada) \\
\hline Jugador 7 & Rotura 5 metatarsiano (grave) \\
\hline Jugador 8 & Principio de pubalgia (moderada)/ 2a lesión principio de pulvalgia (moderada) \\
\hline Jugador 9 & Fractura menisco (grave) \\
\hline
\end{tabular}

1998), un jugador tuvo una lesión leve, 7 jugadores tuvieron lesiones moderadas y tres jugador tuvieron lesiones graves. Según el tipo de lesión, se observaron lesiones de tipo muscular (problemas de espalda y rotura de fibras de aductor), lesiones tendinosas (pubalgia), lesiones articulares (esguince de tobillo y rotura de menisco) y lesiones óseas (edema óseo y fractura quinto metatarsiano). Dos de los nueve jugadores lesionados tuvieron dos lesiones en el periodo de los seis meses de evaluación.

\section{Diferencias en las variables psicológicas} consideradas en función de la ocurrencia o no de lesión deportiva

En la Tabla 2 se muestran diferencias estadísticamente significativas en las dimensiones de regulación emocional, concretamente en percepción emocional $(p=.04)$, gestión auto-emocional $(p=.04)$ y gestión hetero-emocional $(p=.03)$ entre los deportistas lesionados y no lesionados, siendo los deportistas no lesionados los que obtuvieron puntuaciones más elevadas. No se observan diferencias significativas en utilización emocional $(p=29)$.

En relación con las estrategias de afrontamiento utilizadas, se observaron diferencias significativas en restructuración cognitiva $(p=.03)$, conductas de riesgo $(p=.04)$ y retraimiento mental $(p=.04)$. La reestructuración cognitiva era más usada por los no lesionados, mientras que las conductas de riesgo y el retraimiento mental eran más características de los jugadores lesionados. No se observan diferencias significativas en calma emocional $(p=.80)$ y apoyo social $(p=.40)$.

En cuanto a las variables de ansiedad y autoconfianza, se observan diferencias significativas en autoconfianza $(p=$
.04) entre los deportistas lesionados y no lesionados, mostrando mayores puntuaciones en los deportistas carentes de lesión. No se observan diferencias significativas en ansiedad somática $(p=.23)$ y ansiedad cognitva $(p=.26)$.

Respecto a las variables relacionadas con el rendimiento deportivo, se observaron diferencias significativas en control de estrés $(p=.02)$, motivación $(p=.04)$ y habilidad mental $(p=.04)$, siendo los jugadores que no han sufrido lesión los que obtuvieron puntuaciones más elevadas en estas frente a aquellos jugadores que habían experimentado al menos una lesión en ese periodo de tiempo. No se observan diferencias significativas en rendimiento $(p=.69)$ y cohesión $(p=.49)$.

\section{Discusión}

El presente trabajo analiza las posibles variables psicológicas relacionadas con la vulnerabilidad del deportista a padecer una lesión deportiva. Los resultados ponen de manifiesto la importancia de las variables de regulación emocional, y en particular, y como aspecto novedoso del presente trabajo, de la gestión emocional. La ansiedad no parece desempeñar un papel significativo, mientras que la autoconfianza, como variable positiva se encuentra asociada a la no ocurrencia de lesión. Finalmente, el control del estrés y ciertas estrategias de coping en particular, así como la motivación y la habilidad mental desempeñan un papel relevante en la prevención de la lesión deportiva. Estos resultados, confirman las hipótesis previamente planteadas.

La literatura previa coincide en el papel que los recursos de afrontamiento del deportista desempeñan en la lesión deportiva (Garcia-Mas et al., 2014). Estos recursos hacen referencia al conjunto de estrategias tanto cognitivas como 
Tabla 2. Diferencias psicológicas (CSAI-2, CPRD, ACSQ-1 e IE) entre lesionados $(n=9)$ y no lesionados ( $n=13$ ) utilizando la prueba no paramétrica U de Mann-Whitney.

\begin{tabular}{|c|c|c|c|}
\hline & $\begin{array}{c}\text { No lesionado } \\
\text { Media (D.T.) } \\
\quad(n=13)\end{array}$ & $\begin{array}{l}\text { Lesionado } \\
\text { Media (D.T.) } \\
\quad(n=9)\end{array}$ & $\mathrm{U}(p)$ \\
\hline \multicolumn{4}{|l|}{ Inteligencia emocional: } \\
\hline Percepción emocional & $4.25(.49)$ & $3.57(.82)$ & $21.50(.04)$ \\
\hline Gestión auto-emocional & $4.09(.64)$ & $3.54(.55)$ & $21.00(.04)$ \\
\hline Gestión hetero-emocional & $4.12(.55)$ & $3.26(.87)$ & $19.00(.03)$ \\
\hline Utilización emocional & $4.11(.69)$ & $4.10(.94)$ & $34.00(.29)$ \\
\hline \multicolumn{4}{|l|}{ Afrontamiento deportivo: } \\
\hline Calma emocional & $3.97(.47)$ & $4.01(.55)$ & $27.00(.80)$ \\
\hline Reestructuración cognitiva & $4.08(.43)$ & $3.58(.87)$ & $19.00(.03)$ \\
\hline Retraimiento mental & $2.01(.65)$ & $2.55(.67)$ & $21.00(.04)$ \\
\hline Conductas de riesgo & $2.73(.63)$ & $3.29(.69)$ & $19.00(.04)$ \\
\hline Apoyo social & $2.80(.64)$ & $2.90(.60)$ & $36-00(.40)$ \\
\hline \multicolumn{4}{|l|}{ Ansiedad y autoconfianza: } \\
\hline Ansiedad somática & 2.04(.53) & $2.09(.45)$ & $33.00(.26)$ \\
\hline Ansiedad cognitiva & $2.22(.49)$ & $2.25(.54)$ & $31.00(.23)$ \\
\hline Autoconfianza & $3.43(.37)$ & $3.00(.51)$ & $19.00(.04)$ \\
\hline \multicolumn{4}{|c|}{$\begin{array}{l}\text { Variables relacionadas con el } \\
\text { rendimiento deportivo: }\end{array}$} \\
\hline Control del estrés & $50.06(11.31)$ & $39.66(10.21)$ & $18.50(.02)$ \\
\hline Rendimiento & $17.27(6.92)$ & 17.88(5.73) & $42.00(.69)$ \\
\hline Motivación & 25.12(3.13) & $20.66(5.42)$ & $21.50(.04)$ \\
\hline Habilidad mental & $26.87(5.55)$ & $22.33(5.27)$ & $21.00(.04)$ \\
\hline Cohesión & 21.68(3.80) & $21.22(4.76)$ & $38.00(.49)$ \\
\hline
\end{tabular}

conductuales que los deportistas utilizan para gestionar demandas internas o externas que sean percibidas como excesivas para los recursos que posee el individuo (Lazarus y Folkman, 1984). Los resultados obtenidos parecen indicar que los deportistas que no presentan lesión hacen uso de estrategias cognitivas orientadas al problema (relacionadas con procesos perceptivos) mientras que los deportistas que tienen lesión utilizan estrategias evitativas y asumen más riesgos. Intuitivamente, podría atribuirse una mayor posibilidad de lesión en los jugadores que llevan a cabo conductas de riesgo en el deporte, entre ellas no calentar correctamente o no usar protecciones adecuadas, competir pese a las molestias y el dolor o exhibir conductas excesivamente agresivas (del Valle Soto et al., 2018). Igualmente, conviene señalar que la limitación de recursos personales ante situaciones estresantes contribuye al incremento de probabilidad de lesión (Diaz, 2001; Sánchez-Beleña y García-Naveira, 2017).

La capacidad de afrontar estas situaciones no se refiere solo a la resolución práctica de los problemas, sino también a la capacidad de la gestión de las emociones y al control del estrés frente a la situación-problema. En este contexto, entendemos la gestión emocional como la capacidad para identificar y valorar las emociones (percepción emocional) así como de expresar y regular las emociones de uno mismo (gestión auto-emocional) y las de los demás (gestión hetero-emocional) (García et al., 2013). Aunque algunos estudios señalan la estabilidad y la calma emocional como variables asociadas a los deportistas con un menor número de lesiones (Berengüí et al., 2013; Rubio et al., 2014), ningún estudio, desde nuestro conocimiento, ha contemplado el papel de la gestión hetero-emocional en relación con la lesión. Parece 
que los deportistas con una mejor gestión de las emociones de los demás serían menos vulnerables a tener lesión. Ante la ausencia ya señalada de trabajos previos en esta línea, podría aludirse al hecho de que algunos trabajos ya han observado que las personas que llevan a cabo gestos de ayuda, de contribución a otras personas, experimentan un aumento de oxitocina (Milillo, 2014). La secreción de oxitocina mejora la afectividad positiva, contribuyendo a disminuir los niveles de estrés (Rivas et al., 2012). El hecho de que disminuyan los niveles de cortisol (hormona del estrés) podría favorecer la recuperación de los músculos y como consecuencia, disminuir la probabilidad de lesionarse. De esta forma la gestión hetero-emocional actuaría como protector de la salud a través de la reducción de los niveles de estrés (Buñuel, 2019).

En lo que se refiere al control del estrés, éste hace referencia a la capacidad de respuesta del deportista para hacer frente a los estímulos potencialmente demandantes del entrenamiento y/o competición, caracterizadas por la necesidad de control (Campos et al., 2017; González-Campos et al., 2015). Los resultados del presente trabajo ponen de manifiesto que los deportistas no lesionados controlan mejor el estrés que los lesionados. Diferentes autores han encontrado evidencias respecto a la relación entre la lesión deportiva y los diferentes aspectos que pueden englobarse dentro del control de estrés, como es el caso de la capacidad de concentración (Kerr y Minden, 1988), el manejo de la capacidad atencional en situaciones de entrenamiento y competición (Olmedilla et al., 2006), y unos niveles reducidos de tensión (Olmedilla et al., 2018; Olmedilla et al., 2014), todos ellos asociados con un menor riesgo de lesión deportiva.

La autoconfianza implica la autopercepción de competencias asociadas al éxito deportivo (Vealey, 2009) y conlleva anticipaciones cognitivas de objetivos y logros (Berenguií y Puga, 2015). La experiencia, los estados fisiológicos y emocionales determinan la certeza que tiene el deportista de su propia capacidad (Hernandez-Mendo, 2006). Existen diferentes investigaciones previas que aportan resultados significativos en la línea de lo obtenido en este estudio, encontrando que bajos niveles de autoconfianza son predictores significativos de la lesión en futbolistas (Ivarsson, 2008; Johnson y Ivarsson, 2011). En esta misma línea, Abenza et al., (2009) señalan que un mayor riesgo a padecer lesiones estaba asociado con valores medios y bajos en autoconfianza, y otros trabajos (Berengüí et al., 2011; Berengüí y Puga, 2015) encuentran una correlación significativa negativa entre esta variable y el número de lesiones. Gimeno et al. (2007), por su parte, añaden que la baja autoconfianza se relaciona con una mayor incidencia de lesiones por sobrecarga.

Parece, por lo tanto, que la evaluación positiva del deportista de sus propias habilidades, así como de su capacidad de actuar con seguridad y mantener estable la creencia sobre sus capacidades personales, es indispensable para desarrollar la técnica y táctica adecuada y adaptada a la situación, derivando, como consecuencia, en un menor riesgo de lesión deportiva (Berengüí, 2011).

La principal limitación de este trabajo a tener en cuenta a la hora de interpretar los resultados es que el estudio es de tipo transversal, la muestra de la que partimos es pequeña, está constituida únicamente por hombres, no se han contemplado los efectos de las lesiones previas en las variables psicológicas evaluadas y tampoco se ha podido estudiar diferencias según la gravedad de las lesiones. Finalmente, conviene señalar que se necesitan más estudios al respecto, ampliar la muestra, con otras categorías deportivas, deportes, grupos de edad, analizar las diferencias de sexo, etc., para poder valorar la generalización de estos resultados a otros grupos sociodemográficos.

\section{Aplicaciones prácticas}

Los resultados obtenidos conllevan importantes implicaciones prácticas. Generalmente, cuando se trata de prevenir lesiones, las medidas que se llevan a cabo se relacionan con la mejora de las instalaciones, haciendo uso de equipos protectores, o con la optimización de la preparación física (Brewer, 2009). No obstante, adicionalmente a las medidas habitualmente empleadas, y a la vista de los resultados obtenidos en este estudio, resulta imprescindible la necesidad de trabajar sobre las variables y estrategias psicológicas del deportista para conseguir una menor vulnerabilidad a la lesión.

Los resultados obtenidos muestran que el uso de estrategias de afrontamiento adaptativas como la reestructuración cognitiva, una buena gestión emocional y un buen control del estrés se asocian a los deportistas no lesionados. Teniendo en cuenta estas características, sería interesante diseñar programas de intervención psicológica basados en la adquisición y mejora de estas habilidades. Se ha demostrado que la formación en técnicas cognitivas, biofeedback, relajación, visualización guiada, o control atencional, entre otras, son valiosas a la hora de dotar al deportista de recursos de afrontamiento para el control del estrés, así como en la reducción de lesiones, en mayor o menor medida (Williams y Andersen, 2007). Igualmente, el fomento de competencias vinculadas al establecimiento de objetivos y expectativas realistas, a la regulación de pensamientos desadaptativos y a la retroalimentación sobre resultados pueden contribuir al bienestar del deportista (Olmedilla y Dominguez-Igual, 2016). Finalmente, otra de las implicaciones prácticas esta- 
ría vinculada a la formación en competencias psicológicas de los técnicos deportivos, concretamente en su estilo de liderazgo y su filosofía de trabajo por su posible influencia en la aparición de la lesión.

\section{Referencias}

Abenza, L., Olmedilla, A., Ortega, E. y Esparza, F. (2009). Lesiones y factores psicológicos en futbolistas juveniles. Archivos de Medicina del Deporte, 26(132), 280-288.

Álvarez, M. D. A. (2003). Programa de entrenamiento psicológico y metodología de trabajo en un equipo de fútbol juvenil. Cuadernos de Psicología del Deporte, 3(2), 83-94.

Andersen, M. B. y Williams, J. M. (1988). A model of stress and athletic injury: Prediction and prevention. Journal of Sport and Exercise Psychology, 10(3), 294-306. https://doi.org/10.1123/ isep. 10.3.294

Berengüí, R. (2011). Personalidad, características psicológicas y lesiones en el deporte de competición: Análisis de relaciones e incidencia en jóvenes deportistas [Tesis Doctoral no publicada]. Universidad de Murcia, Murcia.

Berengüí, R., Brewer, B. W. y Garcés de Los Fayos, E. J. (2013). Aspectos psicológicos en las lesiones deportivas. En F. Arbinaga y E. Cantón (Eds.), Psicología del Deporte y la Salud: Una relación compleja (pp. 197-244). EOS.

Berengüí, R., López-Gullón, J. M., Garcés de Los Fayos, E. J. y Almarcha, J. (2011). Factores psicológicos y lesiones deportivas en lucha olímpica y taekwondo. Revista de Ciencias del Deporte, 7, 91-98.

Berengüí, R. y Puga, J. L. (2015). Predictores psicológicos de lesión en jóvenes deportistas. Revista Costarricense de Psicología, 34(2), 113-129.

Brewer, B. W. (Ed.) (2009). Injury prevention and rehabilitation. En Autor, Sport Psychology. Handbook of Sports Medicine and Science (Cap. 8, pp.75-86). Wiley-Blackwell. https://doi. org/10.1002/9781444303650.ch8

Buceta, J. M. (2008, 3 y 4 Diciembre). Factores psicológicos y lesión: prevención y recuperación. I Simposium Nacional de Psicología del Deporte, Lesiones deportivas, prevención y rehabilitación. Murcia: Universidad Católica San Antonio de Murcia.

Buceta, J. M. (1996). Psicología y lesiones deportivas: prevención y recuperación. Librería-Editorial Dykinson.

Buñuel, P. S. L. (2019). Las emociones: necesidad de su programación para una actividad física más saludable. E-motion: Revista de Educación, Motricidad e Investigación, 13, 59-81. http://dx.doi. org/10.33776/remo.v0i13.3801

Campos, G. G., Valdivia-Moral, P., Zagalaz, J. C., Ortega, F. Z. y Romero, O. (2017). Influencia del control del estrés en el rendimiento deportivo: la autoconfianza, la ansiedad y la concentración en deportistas. RETOS. Nuevas Tendencias en Educación Física, Deporte y Recreación, 32, 3-6.

Capdevila, L. (1997). Metodología de evaluación en psicología del deporte. Psicología del Deporte. Síntesis.

Cozby, P. C. y Pineda, L. (2005). Métodos de investigación del comportamiento. McGraw-Hill Interamericana.
Díaz, P. (2001). Estrés y prevención de lesiones [Tesis Doctoral no publicada]. Universidad Nacional de Educación a Distancia, Madrid.

Eils, E., Streyl, M., Linnenbecker, S., Thorwesten, L., Völker, K. y Rosenbaum, D. (2004). Characteristic plantar pressure distribution patterns during soccer-specific movements. The American Journal of Sports Medicine, 32(1), 140-145. https://doi, org/10.1177/0363546503258932

Fuller, C. W. (2005). An assessment of the relationship between behaviour and injury in the workplace: A case study in professional football. Safety Science, 43(4), 213-224. https://doi.org/10.1016/i.ssci.2005.05.002

Garcia-Mas, A., Pujals, C., Fuster-Parra, P., Núñez, A. y Rubio, V. (2014). Determinación de las variables psicológicas y deportivas relevantes a las lesiones deportivas: Un análisis bayesiano. Revista de Psicología del Deporte, 23(2), 423-429.

García, R. L. y Más, A. G. (2011). Percepción del dolor y fatiga en relación con el estado de ánimo. Cuadernos de Psicología del Deporte, 17(2), 93-106.

García, V., Graupera, J. L., Ruiz, L. M. y Palomo, M. (2013). Inteligencia emocional en el deporte: Validación española del Schutte self Report Inventory (SSRI) en deportistas españoles. Cuadernos de psicología del deporte, 13(1), 25-36. https:// doi.org/10.4321/S1578-84232013000100004

Gimeno, F., Buceta, J. M. y Pérez-Llanta, M. D. C. (2001). El cuestionario «Características Psicológicas Relacionadas con el Rendimiento Deportivo» (CPRD): Características psicométricas. Análise Psicológica, 19(1), 93-113. https://doi.org/10.14417/ap.346

Gimeno, F., Buceta, J. M. y Pérez-Llantada, M. C. (2007). Influencia de las variables psicológicas en el deporte de competición: evaluación mediante el cuestionario Características psicológicas relacionadas con el rendimiento deportivo. Psicothema, 19(4), 667-672.

González-Campos, G., Valdivia-Moral, P., Zagalaz, M. L. y Romero, S. (2015). La autoconfianza y el control del estrés en futbolistas: Revisión de Estudios. Revista Iberoamericana de Psicología del Ejercicio y el Deporte, 10(1), 95-101.

González-Reyes, A., Moo, J. y Olmedilla, A. (2017). Características psicológicas que influyen en las lesiones deportivas de triatletas amateurs de Yucatán, México. Revista de Psicología del Deporte, 26(2), 71-77.

Herring, S.A., Kibler, W. B. y Putukian, M. (2017) Psychological Issues Related to IIIness and Injury in Athletes and the Team Physician: a consensus statement. Current Sports Medicine Reports, 16(3), 189-201

Hinrichs, T. H. (1995) Lesiones deportivas. Hispano Europea. IBM. (2012). IBM SPSS statistics for Windows, (version 21.0). IBM. Ivarsson, A. (2008). Psychological Predictors of Sport Injuries among Soccer Players. University of Halmstad.

Johnson, U. y Ivarsson, A. (2011). Psychological predictors of sport injuries among junior soccer players. Scandinavian Journal of Medicine and Science in Sport, 21, 129-136. https://doi.or$\mathrm{g} / 10.1111 / \mathrm{j} .1600-0838.2009 .01057 . x$

Kerr, G. y Minden, H. (1988). Psychological factors related to the occurrence of athletic injuries. Journal of Sport and Exercise Psychology, 10(2), 167-173. https://doi.org/10.1123/isep.10.2.167

Kerr, J. H., Aub, C. K. F. y Lindner, K. J. (2004). Motivation and level of risk in male and female recreational sport participation. 
Personality and Individual Differences, 37(6), 1245-1253. https:// doi.org/10.1016/i.paid.2003.12.008

Kim, M. S., Duda, J. L. y Ntoumanis, N. (2003). Examination of the Validity and Reliability of the Korean Approach to Coping in Sport Questionnaire (ACSQ-Korean). International Journal of Applied Sports Sciences, 15(1), 36-55.

Lazarus, R. S., y Folkman, S. (1984). Coping and adaptation. En W. D. Gentry (Ed.), The Handbook of Behavioral Medicine (pp. 282325). Guilford.

Llana, S., Pérez, P. y Lledó, E. (2010). La epidemiología en el fútbol: una revisión sistemática. Revista Internacional de Medicina y Ciencias de la Actividad Física y del Deporte/International Journal of Medicine and Science of Physical Activity and Sport, 10(37), 22-40.

Martens, R., Burton, D., Vealey, R. S., Bump, L. A. y Smith, D. E. (1990). Development and validation of the competitive state anxiety inventory-2. En R. Matens, R. S. Vealey y D. Burton (Eds.) Competitive anxiety in sport (pp. 117- 190). Human Kinetics.

Hernández- Mendo, A. (2006). Un cuestionario para la evaluación psicológica de la ejecución deportiva: estudio complementario entre TCT y TRI. Revista de Psicología del Deporte, 15(1), 71-93.

Milillo, L. S. (2014). Reseña bibliográfica de" La molécula de la felicidad". Filosofía de la Economía, 3(1), 121-125.

Mugele, H., Plummer, A., Steffen, K., Stoll, J., Mayer, F. y Müller, J. (2019). Programa de Prevención de Lesiones Específico del Deporte versus General en Deportistas: Una Revisión Sistemática del Efecto sobre los Índices de Lesiones-Ciencias del Ejercicio. Revista de Entrenamiento Deportivo, 33(3).

Olmedilla, A., García-Alarcón, M. y Ortega, E. (2018). Relaciones entre lesiones deportivas y estrés en fútbol 11 y fútbol sala femenino. Journal of Sport \& Health Research, 10(3), 339-348.

Olmedilla, A., García-Montalvo, C. y Martínez-Sánchez, F. (2006). Factores psicológicos y vulnerabilidad a las lesiones deportivas: un estudio con futbolistas. Revista de Psicología del Deporte, $15(1), 37-52$.

Olmedilla, A., Laguna, M. y Blas, A. B. (2011). Lesiones y características psicológicas en jugadores de balonmano. Revista Andaluza de Medicina del Deporte, 4(1), 6- 12.

Olmedilla, A., Ortega, E. y Gómez, J. M. (2014). Influencia de la lesión deportiva en los cambios del estado de ánimo y de la ansiedad precompetitiva en futbolistas. Cuadernos de Psicología del Deporte, 14(1), 55-62. https://doi.org/10.4321/S157884232014000100007

Olmedilla, A. y Dominguez-Igual, J. J. (2016). Entrenamiento psicológico para la mejora de la atención y la autoconfianza en un futbolista. Revista de Psicología Aplicada al Deporte y al Ejercicio Físico, 1(1), Artículo e4 https://doi.org/10.5093/rpadef2016a4

Olmedilla, A. y García-Mas, A. (2009). El modelo global psicológico de las lesiones deportivas. Acción Psicológica, 6(2), 77-91.

Padegimas, E. M., Stepan, J. G., Stoker, G. E., Polites, G. M. y Brophy, R. H. (2016). Epidemiology and Severity of Sports and Recreation Injuries Presenting to a Tertiary Adult Emergency Department. The Physician and Sports Medicine, 44(3), 263-268. https://doi.org/10.1080/00913847.2016.1171683

Palmi, J. (2014). Psicología y lesión deportiva: Una breve introducción. Revista de Psicología del Deporte, 23(2), 389-393.
Pascual, A. y Aragües, G. M. (1998). Lesiones deportivas y rasgos de ansiedad en los jugadores de fútbol. Medicina Clínica, 17 (2), 45-48.

Prieto, J. M., Labisa, A. y Olmedilla, A. (2015). Ansiedad competitiva, competitividad y vulnerabilidad a la lesión deportiva: perfiles de riesgo. Revista Iberoamericana de Psicología del Ejercicio y el Deporte, 10(2), 293-300.

Prieto, J. P. y Olmedilla, A. (2015). Ansiedad Competitiva, Competitividad y Vulnerabilidad a la Lesión Deportiva. Revista Iberoamericana de Psicología del Ejercicio y el Deporte, 10(2), 293-300.

Pujals, C., Rubio, V. J., Márquez, M. O., Iglesias, I. S. y Ruiz-Barquín, R. (2016). Estudio epidemiológico comparativo sobre lesiones deportivas en una muestra española de 25 distintos deportes. Revista de Psicologia del Deporte, 25(2), 271-279.

Ríos, J., Pérez, Y., Fuentes, E. y De Armas, M. (2019). Efecto de las lesiones deportivas sobre variables psicológicas en lanzadores de Béisbol. Revista de Ciencia y Tecnología en la Cultura Física, 14(3), 403-415.

Rivas, C., Romero, A., Pérez-Llantada, M. D. C., López de la Llave, A., Pourtau, M., Molina, I., González, J. y Garcia-Mas, A. (2012). Bienestar psicológico, salud general, autonomía percibida y lesiones en futbolistas. Revista de Psicología del Deporte, 21(2), 365-371.

Rozen, W. M. y Horne, D. J. L. (2007). The Association of Psychological Factors with Injury. Incidence and Outcome in the Australian Football League. Individual Differences, 5(1), 73-80.

Rubio, V. J., Pujals, C., de la Vega, R., Aguado, D. y Hernández, J. M. (2014). Autoeficacia y lesiones deportivas: ¿factor protector o de riesgo? Revista de Psicología del Deporte, 23(2), 439-444.

Sánchez-Beleña, F. y García-Naveira, A. (2017). Sobreentrenamiento y deporte desde una perspectiva psicológica: estado de la cuestión. Revista de Psicología Aplicada al Deporte y al Ejercicio Físico, 2(12), Artículo e12 https//doi.org/10.5093/rpade$\underline{\mathrm{f} 2017 \mathrm{a} 8}$

Schinke, R.J., Stambulova, N.B., Sic. G. y Moore, Z. (2017). International society of sport psychology position stand: Athletes' mental health, performance, and development. International Journal of Sport and Exercise Psychology, 16(6), 1-18. https://doi. org/10.1080/1612197X.2017.1295557

Schutte, N. S., Malouff, J. M., Hall, L. E., Haggerty, D. J., Cooper, J. T., Golden, C. J. y Dornheim, L. (1998). Development and validation of a measure of emotional intelligence. Personality and Individual Differences, 25(2), 167-177. https://doi.org/10.1016/ S0191-8869(98)00001-4

Soligard, T., Schwellnus, M., Alonso, J. M., Bahr, R., Clarsen, B., Dijkstra, H.P., Gabbett, T., Gleeson, M., Hägglund, M., Hutchinson, M. R., van Rensburg, C. J., Khan, K. M., Meeusen, R., Orchard, J. W. Pluim, B. M., Raftery, M., Budgett, R. y Engebretsen, L. (2016). How much is too much? (Part 1) International Olympic Committee consensus statement on load in sport and risk of injury. British Journal of Sports Medicine. 50(17), 1030-1041. https://doi.org/10.1136/bjsports-2016-096581

Tscholl, P., O'Riordan, D., Fuller, C. W., Dvorak, J., Gutzwiller, F. y Junge, A. (2007). Causation of injuries in female football players in top-level tournaments. British journal of sports medicine, 41(1), 8-14.

Turner, C., McClure, R. y Pirozzo, S. (2004). Injury and risk-taking behavior a systematic review. Accident Analysis \& Prevention, 36(1), 93-101. https://doi.org/10.1016/S0001-4575(02)00131-8 
Vealey, R. S. (2009). Con dence in sport. En B.W. Brewer (Ed.), Sport Psychology. John Wiley \& Sons. https://doi. org/10.1002/9781444303650.ch5

Williams, J. M. y Andersen, M. B. (2007). Psychosocial antecedents of sport injury and interventions for risk reduction. En G. Tenenbaum y R. C. Eklund (Eds.), Handbook of sport psychology (pp. 379-403). John Wiley \& Sons Inc.

Zurita, F., Fernández, R., Cachón, J., Linares, D. y Pérez, A. J. (2014). Aspectos psicosomáticos implicados en las lesiones deporti- vas. Cuadernos de Psicología del Deporte, 14(2), 81-88. https:// doi.org/10.4321/S1578-84232014000200009

Zurita, F. Z., Fernández, S. R., Extremera, M. O., Sánchez, M. C., Cuberos, R. C. y González, M. D. M. C. (2017). Análisis de la resiliencia, ansiedad y lesión deportiva en fútbol según el nivel competitivo. Cultura, ciencia y deporte: Revista de ciencias de la actividad física y del deporte de la Universidad Católica de San Antonio, 12(35), 135-142. 